\title{
Development of vascular disease models to explore disease causation and pathomechanisms of rare vascular diseases
}

\author{
Rebecca L. Harper ${ }^{1} \cdot$ Elisa A. Ferrante ${ }^{1} \cdot$ Manfred Boehm $^{1}$ (D)
}

Received: 27 January 2022 / Accepted: 10 February 2022 / Published online: 1 March 2022

This is a U.S. government work and not under copyright protection in the U.S.; foreign copyright protection may apply 2022

\begin{abstract}
As the field of medicine is striving forward heralded by a new era of next-generation sequencing (NGS) and integrated technologies such as bioprinting and biological material development, the utility of rare monogenetic vascular disease modeling in this landscape is starting to emerge. With their genetic simplicity and broader applicability, these patient-specific models are at the forefront of modern personalized medicine. As a collective, rare diseases are a significant burden on global healthcare systems, and rare vascular diseases make up a significant proportion of this. High costs are due to a lengthy diagnostic process, affecting all ages from infants to adults, as well as the severity and chronic nature of the disease. Their complex nature requires sophisticated disease models and integrated approaches involving multidisciplinary teams. Here, we review these emerging vascular disease models, how they contribute to our understanding of the pathomechanisms in rare vascular diseases and provide useful platforms for therapeutic discovery.
\end{abstract}

Keywords Rare vascular disease modeling $\cdot$ Monogenetic vascular disease $\cdot$ iPSC $\cdot$ Endothelia cell $\cdot$ Smooth muscle cell · Fibroblast · Vascular organoid

\section{Introduction}

Rare diseases make up a significant proportion of the global disease burden. There are between 6,000 and 8,000 identified rare diseases and the majority is caused by a mutation in a single gene. These monogenetic diseases tend to be severe in phenotype causing significant debilitation with decreased quality of life and are typically life-limiting with three-quarters affecting children [1]. Most rare diseases have no treatment or cure and are often diagnosed (if at all) following a long and expensive investigation. Despite their individual rarity, the chronic and disabling nature of these diseases disproportionality consumes healthcare budgets [2]. Cardiovascular disease (CVD) is the leading cause of mortality globally [3-5] and within this group, there are a

This article is a contribution to the special issue on: Inflammation in vascular diseases - Guest Editors: Mariana Kaplan \& Peter Grayson

Manfred Boehm

boehmm@nhlbi.nih.gov

1 Translational Vascular Medicine Branch, National Heart, Lung and Blood Institute, National Institutes of Health, Bethesda, MD, USA myriad of rare vascular diseases that when combined, make up a significant proportion this burden. In this era of modern medicine, with access to technologies that can assess whole genomes, transcriptomes, biomes, and proteomes to accelerate biological discovery, the development and use of patient-specific disease models is an innovative and increasingly powerful tool to study rare monogenetic vascular diseases. This approach not only addresses the elusive clinical challenges for these rare diseases but also allows for in-depth investigation of disease-causing molecular mechanisms and phenotypes shared with more common vascular disease. Thus, broadening the application of knowledge sourced from rare vascular disease patients to a significant proportion of the global population affected by CVD.

Patient-specific vascular disease modeling draws upon innovative research technologies and pairs this with novel strategies of application (Fig. 1). With the development of unbiased next-generation DNA sequencing (NGS), more specifically whole-exome sequencing (WES) in 2010 and whole-genome sequencing (WGS) in 2009, identification of genes with disease-causing alterations in rare disorders has significantly increased $[1,6,7]$. It is now possible to address the challenges that arise from the heterogeneous 


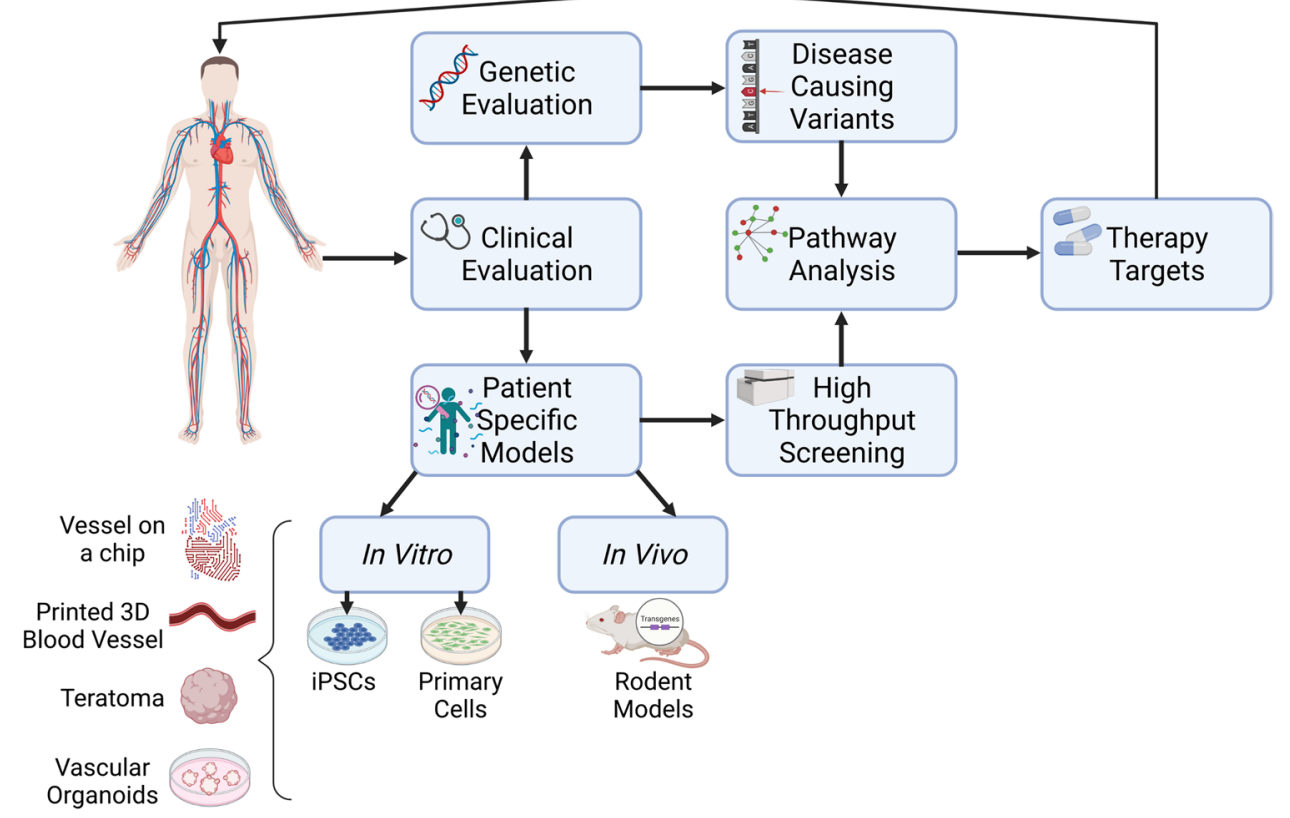

Fig. 1 Patient-specific translational disease modeling pipeline. Starting at the point of the patient, a clinical evaluation is made to ascertain the pathophysiology, severity, and baselines of the patient's condition. Samples are then taken for NGS evaluation where diseasecausing variants can be identified, and novel molecular pathways are discovered, leading to the identification of new therapeutic targets.

pathophysiology of rare vascular diseases, in a more efficient manner [8]. These studies can be extended to assess affected patients to include unaffected relatives and aid in generating a list of candidate targets based on deleteriousness, inheritance patterns, and population frequency [9]. Once the disease-causing gene mutation is identified in a patient, in vitro systems such as induced pluripotent stem cells (iPSCs) and primary cell lines, specifically fibroblasts from skin punches that are relatively easy to obtain, can be used in advanced vascular disease model platforms including vascular organoids (VOs), 3D printed blood vessel constructs, teratomas, and vascular grafts and patches for in vivo transplantation. In addition to in vivo disease models including transgenic mouse models, both approaches can be stably developed to aid further investigation of pathomechanisms. Importantly, these provide a relevant platform for high-throughput drug screening with the ultimate focus on meeting a critical unmet clinical need.

Patient-specific vascular disease models can be used in vitro, in vivo, or ex vivo, developed with the aim to recapitulate and recreate disease phenotypes. In vitro systems employ primary cells derived from a myriad of patient biospecimens, mesenchymal stem cells (MSCs), iPSCs, non-vascular cells, and blood-derived immune
In parallel to this, patient samples are also taken to establish both in vitro and in vivo models. These can be used to further investigate molecular pathways, be used for high-throughput screening, and contribute to proof-of-concept therapy intervention studies. This pipeline is geared to feedback therapy options to the patient with the hope to relieve or even cure the disease. Created with BioRender.com

cells and progenitor cells [10]. These models range from simple two-dimensional monocultures and co-cultures to complex spherical organoids and four-dimensional vascular structures. While in vitro models have significant advantages due to their inexpensive simplicity and potentially unlimited or abundant supply and malleable qualities, they also have shortcomings due to their lack of in vivo environmental factors [3]. In vivo vascular models typically involve rodent models due to their availability, easy manipulation of their genome, accessibility, and cost. However, physiological differences in the cardiovascular systems of mice and rat models make it challenging to translate findings to human disease. Larger animal models such as pigs, sheep, and non-human primates are seen as a preferred alternative to address these differences and their development makes them more applicable to human disease as pre-clinical models [3]. Due to the limitations of both in vitro and in vivo models, some research programs have concentrated on studying ex vivo tissue directly. These models typically use blood vessels isolated from animals or ex planted human tissue to study blood vessels without compromising their architecture [11]. While all these models have their advantages and disadvantages, used in the correct context, they are extremely useful tools for researchers. Using these systems specifically 
for modeling monogenetic vascular diseases is the very basis of personalized and precision medicine, leading to enhanced and accelerated learning.

\section{Vascular disease models}

\section{In vitro vascular model systems}

In the pursuit of developing vascular models that recapitulate human disease, researchers have moved towards in vitro cell models as more a robust, reproducible system to delve into not only disease causation but also pathomechanisms and drug discovery. The simplicity and cost effectiveness of in vitro cell models make them an excellent candidate for rare vascular modeling, and when these models are combined with current NGS technology and cutting-edge platforms, in vitro cell models become an extremely powerful tool.

\section{Primary cells}

The complexity within the cardiovascular system gives rise to many cell types that are individually heterogenous. This array of cells can be dependent on the organ, adjacent cells, vessel structure, and environmental factors such as shear stress, metabolism, gas exchange, and blood derivatives. Thus, it has become imperative to seek cells that originate from the environment that is most relevant to characterize the disease pathology. Primary cells isolated from human and animal tissues have become a staple for in vitro cell model systems. Vascular primary cell in vitro models typically use cardiomyocytes (CMs) and non-myocyte cells to study the heart. Endothelial (EC) and smooth muscle cells (SMCs) are the critical cell types in blood vessels investigations [12] and, further, there are a myriad of other relevant cells that either interact or influence these vascular cells, such as fibroblasts and immune cells.

Cultured primary fibroblasts have proven to be a useful and adaptable tool for investigating rare monogenetic vascular diseases as these cells have been shown to recapitulate disease phenotypes. St Hilaire et al. used primary fibroblasts to identify ecto-5-prime-nucleotidase (NT5E) mutations in patients with arterial calcifications due to deficiency of CD73 (ACDC) and went on to use these primary cells to further characterize loss-of-function of CD73, the protein NT5E encodes [13]. CD73 is an enzyme that converts extracellular AMP to adenosine and inorganic phosphate. A total of 9 patients from 3 separate families were identified with severe calcifications of the lower-extremity arteries and hand and foot capsules. The differentiating pathological feature of ACDC from traditional atherosclerotic vascular calcifications is the calcification and dysplasia occurring in the medial portion of the arterial blood vessel wall. This results in claudication of the calves, thighs, and buttocks, with the patients experiencing chronic and debilitating ischemic pain in their extremities. Fibroblasts from one family showed markedly reduced NT5E RNA, CD73 protein expression, and enzyme activity. These studies also showed increased alkaline phosphatase levels and accumulation of calcium phosphate crystals, all hallmark features of ACDC. Genetic rescue studies and adenonsine treatment reduces alkaline phosphatse and calcium accumulation. Subsequent studies using ACDC patient-specific fibroblasts have shown a role of tissue non-specific alkaline phosphatase (TNAP) as an important mediator for pathological ectopic tissue calcification [14]. Using these patient-specific fibroblasts has provided the impetus for an on-going ACDC clinical trial investigating the utility of the drug Etidronate, which was identified by using patient-specific disease modeling to perform drug screening studies [15]. In parallel to this clinical trial, studies are ongoing using these primary cells, as well as other patient-specific models, to continue to investigate the complex molecular pathomechanisms for patients with ACDC in hopes of finding further effective treatments [14].

Furthermore, primary fibroblasts have played an important role in identifying the importance of hypoxia inducible factor- $1 \alpha$ (HIF1 $\alpha)$ stabilization for angiogenesis and extracellular matrix (ECM) deposition in wound healing of patients with autosomal-dominant hyper IgE syndrome (ADHIES), which is caused by a loss-of-function mutation in STAT3 [16]. Included in the clinical presentation of this disease are immunodeficiency, as well as skeletal, connective tissue and vascular abnormalities, and poor post-infection lung healing, often resulting in pulmonary failure. Using patient-specific skin wound models in parallel with global gene expression analysis of primary fibroblasts, these studies confirmed deficiency in STAT3-controlled transcriptional molecular pathways that controlled ECM remodeling and angiogenic capacity in these patients, with HIF $1 \alpha$ playing a pivotal role in the transcriptional networks. Further studies using these patient fibroblasts confirmed dysregulated angiogenesis and wound healing, as well as helping develop a platform to test and demonstrate the utility of HIF $1 \alpha$ stabilizing drugs, prolyl hydroxylases (PHD) inhibitors, dimethyl fumarate (DMF), and daprodustat. These in vitro studies provided an excellent basis to test the treatment efficacy for further in vivo studies in an AD-HIES mouse model, where the researchers were able to further explore pathomechanisms as well as proof-of-concept to target HIF1 $\alpha$ for therapy in this patient cohort.

In the field of pulmonary arterial hypertension (PAH), primary cells taken from small and large vessels have 
successfully been used to investigate pathomechanisms for this rare fatal disease. While the exact cause and precise pathomechanism remains unclear, there is a causal link between mutations in the bone morphogenetic protein receptor type 2 (BMPR2) and PAH, characterized by increased pulmonary artery pressure (PAP) due to uncontrolled EC and SMC proliferation, resulting in vascular occlusions of distal pulmonary arteries. Primary ECs and SMCs isolated from small, medium, and large vessels of ex vivo transplanted lungs have allowed investigators to identify disease-causing dysfunction within these cell types in isolation, co-culture $[17,18]$, and in systems that recapitulate dynamic shear [19].

Studies such as those described here for ACDC, ADHIES, and PAH have not only helped in elucidating the disease-causing mutations and pathomechanisms of these specific disorders and shown the ability of vascular disease modeling in drug screening for personalized patient-specific therapies but they have also driven the development of valuable platforms that can be adapted and innovated for virtually any other condition, rare or common, that present with vascular calcification, abnormal wound healing, and cellular dysfunction in pulmonary arteries, for example.

While isolation and use of primary cells is possible and preferable to non-vascular immortalized cell lines, the severe phenotypic nature of rare vascular disorders as well as the limited number of candidates can limit the availability and biological diversity of these cell types. It was this limitation that fueled immense interest in circulating progenitor cells. These peripheral blood-derived cells are not only used as biomarkers for endothelial dysfunction [20-22], but they can also be isolated and differentiated into endothelial progenitor cells (EPCs) and smooth muscle progenitor cell (SMPC)like cells and are an ideal patient-specific in vitro model for rare vascular diseases [23]. Mechanistic studies on the proliferative effects of bone morphogenetic protein 9 (BMP9), an agent proposed to be an effective treatment in proof-ofconcept PAH animals models, have shown that PAH-derived EPCs hyper-proliferate in response to BMP9 treatment [24]. These studies have been critical for understanding important molecular and signaling pathways to move towards developing therapies for a fatal rare disease. Endotheliallike progenitor cells have also been used as an effective vehicle for gene therapy in a proof-of-concept PAH animal model, where they were virally transduced with BMPR2 and intravenously given to rats with established PAH [25]. With their affinity to home to certain vascular niches such as areas of trauma and oxidative stress, as well as offering an autologous cell therapy option, these cells are an excellent therapeutic candidate [26, 27]. Despite the increased interest and use of progenitor cells as in vitro vascular models, their differentiation and expansion from such small numbers in the peripheral circulation leads to the loss cell integrity and lifespan, which is limiting to their application. Not to mention ongoing academic debates on progenitor cell characterization, origination, and definition [28-30].

\section{Stem cells}

With the ability to proliferate indefinitely and differentiate into most cell types, stem cells are an extremely useful tool for investigating pathomechanisms of any disease and are the ultimate tool for developing innovative research platforms for disease modeling, therapeutic targeting, high-throughput drug discovery, and regenerative therapies [10].

Induced pluripotent stem cells are a type of stem cell that has been reprogrammed from a somatic cell into a pluripotent cell with the capacity to propagate indefinitely and differentiate into many different cell types, including induced EC (iEC), induced SMC (iSMC), myeloid lineage cells (iMLCs), and induced mesenchymal stromal cells (iMSCs). Importantly, these cells can be derived from peripheral blood mononuclear cells (PBMCs), urine, skin fibroblasts, or keratinocytes from hair. A readily available, relatively easily obtainable cell reservoir coupled with their plasticity makes them a powerful in vitro model for rare vascular diseases. Additionally, once these cells are reprogrammed, they are capable of stably retaining gene mutations [31], making them critical for investigating monogenetic vascular disease [32, 33].

Generation of iPSC lines from rare monogenetic vascular disease is one of the critical components of a patient-specific disease model pipeline. Following NGS studies to identify disease-causing gene variants on patients with unknown disease etiology, the generation of patient-specific iPSC lines to further explore pathomechanisms is an extremely useful tool. Jin et al. demonstrate this by generating iPSC lines from AD-HIES patients carrying a STAT3 mutation [34]. Chen et al. generated iPSC lines from 5 patients with cerebral autosomal-dominant arteriopathy with subcortical infarcts and leukoencephalopathy (CADASIL) and OUTLIN-related autoinflammatory syndrome (ORAS) [32, 33]. Following reprogramming of fibroblasts or PBMCs using a lentivirus system, cells are characterized via immunofluorescence staining, flow cytometry analysis (FACS), and gene expression for their capacity to differentiate into three germ layers: endoderm (AFP), ectoderm (NESTIN), and mesoderm (RUNXI), as well as assessed for their ability to differentiate as a monolayer of cells. Following these functional tests, the lines are karyotyped to ensure the desired gene mutation is retained during the reprogramming. Once the lines are characterized and validated, they have the potential to differentiate into multiple cell types. For all these rare monogenetic diseases, the pathomechanism remains unknown, thus these iPSC patient-specific disease models 
are essential for studying the effect of not only the effect of gene mutations of STAT3, NOTCH3, and OUTLIN but also the role of these genes in normal physiology.

Recently, the capability of generating isogenic controls for patient-specific lines has increased the validity of research using these cells, allowing for off-target effects to be identified and controlled for as well as demonstrating the effect of correcting mutations in specific cell types. The generation of isogenic iPSC controls has emerged from the development of CRISPR/Cas technology, which is an adapted strategy piggybacking off a naturally occurring bacterial gene editing system. The CRISPR/Cas system, using a guide RNA and the Cas enzyme, uses the guide RNA to target a DNA sequence in the genome, where the Cas enzyme can cut the DNA and utilize the cells' own DNA repair machinery to repair the site, incorporating the introduced DNA sequence. Specifically for isogenic iPSC control lines, the site of the patient-specific gene mutation is targeted and removed, and the "non-mutated" sequence is put in its place, generating a patient-specific iPSC control line. Using this strategy, Song et al. created patient-specific isogenic iPSC control to examine pathomechanism in Fabry disease, an X-linked inheritable lysosomal storage disease caused by a deficiency of $\alpha$-galactosidase A (GLA). This leads to an accumulation of globotriaosyceramide (GB3) in ECs of many tissues that can result in multiple organ failure, and dysfunction of endothelial cells in the microvasculature. Using this isogenic iPSC control line to derive ECs, they demonstrated a reversal of inflammatory responses, including decreased cytokine release and specific inflammatory markers such as ICAM, CCL2CCL5, CXCL1, IL6, IL8, monocyte inducible factor, and CXCL10 [35]. The importance of using isogenic controls is highlighted in this monogenetic rare vascular disease model, allowing the examination of pathologic phenotypes in a system without the modeling limitations of using healthy control lines, such as epigenetic, environmental, and genetic background differences.

Endothelial cells are a heterogenous population dependent on the tissue type they originate from and what function they perform in that context. These cells are critically important for regulating tissue homeostasis, regeneration, regulating immune response, and providing a barrier and conduit for molecules. The study of human genotype-phenotype in the context of vascular disease has shown that when dysregulated, the endothelium is pathogenic in many diseases. A critical need has been to establish an in vitro EC platform that can overcome the limited availability of patient-specific ECs. Human iPSC (hiPSC)-derived ECs (hiPSC-ECs) are a powerful tool not only for investigating pathomechanisms but also for drug screening and cell therapy [10]. There have been many reports of varying methods of differentiating hiPSCs into hiPSC-ECs [36, 37] for a myriad of applications. The generated cells will not only express endothelial surface markers: CD31, VE-cadherin, vascular endothelial growth factor (VEGF), and KDR but can also form tubes in Matrigel as well as a barrier monolayer and take-up low density lipoprotein (LDL) [37-39]. Comparison studies between hiPSC-ECs and primary pulmonary arterial endothelial cells (PAECs) isolated from PAH patients with a mutation in BMPR2 showed that hiPSC-ECs showed similar characteristics to the PAEC in adhesions, tube formation, surface marker expression, migration, and survival [40]. HiPSC-ECs have successfully been used to investigate pathomechanisms in familial PAH (FPAH). Gu et al. were able to show reduced adhesion, migration, and angiogenesis potential in patientderived hiPSC-ECs with mutations in BMPR2, compared with healthy control hiPSC-ECs [41]. These mechanistic studies led to further work where they utilized hiPSC-ECs in a high-throughput drug discovery coupled with a phenotypic screening strategy to identify tryphostin-AG1296, a plateletderived growth factor (PDGF) receptor inhibitor, capable of inducing apoptosis and cell migration [42]. The advantage of these hiPSC-EC vascular cell models is their patient specificity and applicability to the disease pathogenesis. These studies were critical in identifying, assessing, and producing an alternative therapy option for FPAH patients. Another research study using hiPSC-ECs to study pathomechanisms in Fabry disease used hiPSC-EC to identify a decrease in superoxide dismutase 2 , increased reactive oxygen species (ROS), and enhanced AMPK activity causing dysregulated EC function. Targeted therapy directed towards this novel pathway may lead to a viable treatment option for those with Fabry disease [43].

Vascular disease not only encompasses EC dysregulation, but SMCs also play an important role in blood vessel formation and maintenance. Human iPSC-derived SMCs (hiPSCSMCs) have been used to investigate this cell type's role in rare vascular diseases. Granata et al. developed a vascular model for Marfan syndrome (MFS) to better understand the pathogenesis of aortic aneurysm, using hiPSC-SMCs [44]. This model was able to recapitulate the phenotype seen in MFS aortas, including defects in fibrillin-1, ECM degradation, transforming growth factor- $\beta$ (TGF- $\beta$ ) signaling, contraction, and apoptosis, as well as to identify a novel pathogenic pathway involving p38 and Kruppel-like factor 4 (KLF4) [44].

Both the hiPSC-EC and hiPSC-SMC in vitro models are particularly useful for gene editing studies where both $\mathrm{Gu}$ et al. and Granata et al. used CRISPR-based gene editing technology to correct the known mutations in these cell models and demonstrate reversal of the disease phenotypes $[41,44]$. In addition to this plasticity in editing iPSCs, the ability to generate many cell types from a single patient allows complex models that recapitulate blood vessel physiology more accurately. Co-culture of hiPSC-ECs with hiPSC-SMCs enables modeling and investigation of 
the crosstalk that exists in vivo between these cell types in blood vessels, increasing the accuracy of the mechanistic evaluations of one or both cell types in the context of vascular disease. Furthermore, the role of hiPSC-MLCs on these cells within this system can also be assessed. Addition of either hiPSC-monocyte or macrophages either activated or not activated to a monolayer or co-culture can provide critical insight into the role of acute or chronic inflammatory processes in the context of vascular disease. These processes are often purported to be a trigger for disease development and progression so the utility of patient-specific iPSC for investigating rare monogenetic vascular diseases is extensive.

The ability to generate patient-specific human iPSCs opens the door for developing cell-based personalized disease modeling for many different cell types. These cells are then used as the foundation for advanced vascular disease model platforms including VO, 3D printed blood vessel constructs, teratomas, and vascular grafts and patches for in vivo transplantation. These platforms are employed to elucidate pathomechanisms and become the medium for high-throughput drug screening in a more physiologically relevant setting. Co-culture systems have been helpful in elucidating molecular crosstalk and manipulating cell signaling between cell types. However, this model is a monolayer system, and we know that blood vessels are tubular, and the alignment of both ECs and SMCs is critical in determining their function within this system so 3D model systems can be used to provide complementary data that the $2 \mathrm{D}$ settings cannot provide. Organ-on-a-chip technology uses a hydrogel in a chamber on microfluidic chips, where iPSC cells are cultured to form 3D blood vessel-like structures. These chips can accommodate multiple cell types, giving rise to a $3 \mathrm{D}$ co-culture system with the goal of recreating an even more physiologically relevant in vitro model that can include flow-induced shear stress [45]. These engineered microvascular models have been used to assess endothelial barrier dysfunction, cancer, organ regeneration, and for drug screening [46]. Printing of 3D blood vessels using iPSCs is another platform emerging to bridge the gap between 2D monoculture and representative in vitro vascular models [47]. While this technology is in its infancy, there have been promising advances for this platform. Zhou et al. developed a 3D vessel printing method that can generate small diameter vessels loaded with vascular SMC and EC and have shown that the cells can proliferate in culture with fluid flow [48]. Their bioprinting method not only allows for variable diameter vessels with distinct cell layers but the fluid flow can also be modulated to recapitulate the physiological shear environments observed in different types of vessels (venules, veins, arterioles, etc.) while enabling cell-to-cell interactions that can be critical for vessel homeostasis. When used in combination with patient-derived primary, iPSC, and iPSC-derived cells, one can hypothesize that this technology may be coupled with next-generation sequencing studies to not only shed light on the unknowns of rare genetic vascular disease but to also test therapeutic approaches in a high-throughput manner that is more physiologically relevant. Further, as these vessels are fine-tuned with these techniques in fluidic tissue culture, they may be then implanted or engrafted into humanized animal models to add the complexities of blood flow, the impact of additional cell types, and the metabolism in genetic or disease mechanism studies.

Cui et al. developed a cardiac patch that not only withstood in vitro physiological testing but was also successfully engrafted into a murine model [49]. This platform is a great method for meeting a clinical need of engraftment of blood vessels for an array of conditions, and an effective vascular disease model platform. As these technologies gain more momentum, when they are coupled with readily available patient-specific iPSCs, they will provide a much needed and accessible model to investigate monogenetic rare vascular diseases.

The development of VOs is a promising advancement on studying the vasculature in the context of specific tissue [50]. While this technology is still in its infancy, its potential and utility for studying rare monogenetic vascular disease is immeasurable. These diseases tend to be multi-facetted with the involvement of many organs and systems, making it a complex problem-solving exercise. In 2019, Wimmer et al. shared, in great detail, a reproducible protocol developed for the generation of self-assembling vascular organoids from human iPSCs, showing that the vasculature formed in these constructs mimicked anatomical and physiological characteristics of the human microvasculature [50]. Further, they implanted these vascular organoids into immunocompromised mice, where they were able to integrate into the murine vasculature and further differentiate into various types of blood vessels. Since this protocol was published, this and other groups have implemented this technology for a myriad of applications, ranging from angiogenesis [51] to pathomechanism investigation and elucidation of signaling pathways/cellular interactions [52-54] as well drug screening in conditions such as type 2 diabetes [55] and, most recently, COVID-19 [56, 57]. As this technology continues to be reproduced, used, and further improved, it opens the doors for personalized, patient-derived disease modeling for a virtually unlimited number of vasculopathies.

The use of iPSC-derived teratoma's for studying vascularization in rare vascular diseases bridges in vitro technology with in vivo animal model utility. Upon subcutaneous injection of patient-specific iPSCs in mice, vascularized teratomas will form and start growing. These are then explanted and can be assessed in a variety of ways to help in elucidating cause and effect of monogenetic diseases on vascular formation. Dmitrieva et al. used this technique to 
assess impaired angiogenesis and altered ECM metabolism in patients with AD-HIES [16]. With this platform, it was confirmed that AD-HIES patients have defective neo-vascularization capacity, which was confirmed by observing decreased teratoma weights and CD31 positive cells after 8 weeks of growth.

In vitro vascular models have advanced considerably in the last 10-15 years. This has been mostly due to the increased knowledge combined with the use of iPSC technology and isolation methods for primary cells. With the introduction of NGS technology, patient-specific vascular modeling employing these techniques and platforms has accelerated our understanding of pathomechanisms and aided in identifying critical therapeutic targets.

\section{In vivo vascular model systems}

Animal models are an important component of rare vascular disease modeling as an up-scalable and readily available alternative to patient-specific in vitro systems, which rely on a limited supply of subjects. Despite the obvious divergence from human anatomy and physiology, the use of animal models remains critical for pathomechanisms investigation and proof-of-concept studies for potential novel therapies. In the context of cardiovascular disease, both large and small animal studies have been insightful for discovering fundamental pathomechanisms as well as assessing pharmaceutical interventions [58]. A recent review of over 9,000 publications utilizing mouse models reported that after a genome-wide association study (GWAS), there was significant overlap (45 of 46 genes) shared between mouse and humans involved in coronary artery disease [59]. The advantage of this over in vitro platforms is the context of a multiorgan, integrated system where a diverse range of environments, treatments, and genetic manipulations can be applied with a plethora of endpoint results that can be assessed. As most rare vascular diseases are monogenetic, animal models and more specifically transgenic mouse models are particularly useful [60].

Mouse models have become by far the most widely used animal models in vascular research. With the development of genetic and molecular manipulation, these models have become somewhat "humanized" [61]. Targeted manipulations developed by individual investigators have given this field the tools needed to narrow their studies on specific organs and cell types, as well as when and how to induce these changes [62]. Specifically in the field of PAH, there are models targeting only EC [63], SMC [64], and, recently, monocytes [65], where inducible knockout of $B M P R 2$ in these cell types is used for either pathomechanistic or therapeutic rescue studies. A critical understanding of the $B M P R 2$ signaling cascade and its components of some mothers against decapentaplegic
(SMAD) and non-SMAD proteins have been extensively studied in the pursuit of more effective treatments [66-71]. A novel transgenic mouse model has led to insightful pathomechanisms in stimulator of interferon genes (STING)associated vasculopathy with onset in infancy (SAVI), an autoinflammatory disorder caused by a gain-of-function mutation in TMEM173 encoding the STING protein [72]. The overactivation of STING leads to a chronic activation of interferon pathways leading to endothelial dysfunction. This disease affects multiple organs and coupled with its genetic component, rarity, and limitations associated with sample collection from infants is a great candidate to investigate using a mouse vascular disease model. Another rare vascular disease that has seen great advancement in understanding and treatment with the aid of mouse models is sickle cell anemia (SCA) $[73,74]$. There are currently many clinical trials for SCA underway that target vasoocclusion, inflammation, gene editing, and cell therapy with gene editing, all made possible through the use of proof-ofconcept animal models [74].

With their genetic simplicity, rare monogenetic vascular diseases are ideal candidates for transgenic mouse models. Despite the known physiological limitations of animal models, they have been proven to show relevant genetic and phenotypic similarities with human disease, particularly following gene modification. Thus, there remains a certain utility for rare vascular disease in vivo models when used in conjunction with patient-specific in vitro platforms. These models are particularly helpful to extensively study pathomechanisms in an integrated multiorgan system as well as for proof-of-concept therapy approaches.

\section{Concluding remarks}

The continued advancement of NGS and the incorporation of cutting-edge technologies such as bioprinting and nanotechnology biomaterial development will continue to revolutionize the landscape for rare vascular disease modeling. This has heralded a new era of complex patient-specific experimental platforms, integrated technologies with the output and analysis of big data with the ultimate goal of seamlessly translating medicine from the bed to bench-side and back again. Personalized medicine is gaining tremendous momentum and rare monogenetic vascular diseases are the perfect candidate. These approaches have far-reaching positive outcomes not only for addressing the enormous disease burden that these conditions have collectively on the global community but also the scientific discoveries that they enable have broader applicability to similar pathomechanisms of more common cardiovascular disease. 
Abbreviations CVD: cardiovascular disease; WES: whole-exome sequencing; WGS: whole-genome sequencing; NGS: next-generation sequencing; MSCs: mesenchymal stem cells; iPSCs: induced pluripotent stem cells; CMs: cardiomyocytes; EC: endothelial cell; SMC: smooth muscle cell; NT5E: ecto-5-prime-nucleotidase; ACDC: arterial calcifications due to deficiency of CD73; TNAP: tissue non-specific alkaline phosphatase; HIF $1 \alpha$ : hypoxia inducible factor- $\alpha 1$; ECM: extracellular matrix; AD-HIES: autosomal-dominant hyper IgE syndrome; PHD: prolyl hydroxylases; DMF: dimethyl fumarate; PAH: pulmonary arterial hypertension; BMPR2: bone morphogenetic protein receptor type 2; PAP: pulmonary artery pressure; EPCs: endothelial progenitor cells; SMPCs: smooth muscle progenitor cells; BMP9: bone morphogenetic protein 9; iEC: induced endothelial cell; iSMC: induced smooth muscle cell; iMLCs: myeloid lineage cells; iMSCs: induced mesenchymal stromal cells; PBMCs: peripheral blood mononuclear cells; CADASIL: cerebral autosomal-dominant arteriopathy with subcortical infarcts andleukoencephalopathy; FACS: flow cytometry analysis; hiPSC: human iPSC; hiPSC-EC: human-induced pluripotent stem cell-derived endothelial cell; PAECs: pulmonary arterial endothelial cells; FPAH: familial pulmonary arterial hypertension; PDGF: plateletderived growth factor; GLA: $\alpha$-galactosidase A; ROS: reactive oxygen species; hiPSC-SMC: human-induced pluripotent stem cell-derived smooth muscle cell; MFS: Marfan syndrome; TGF- $\beta$ : transforming growth factor- $\beta$; KLF4: Kruppel-like factor 4; VOs: vascular organoids; GWAS: genome-wide association study; SMAD: some mothers against decapentaplegic; STING: stimulator of interferon genes; SAVI: stimulator of interferon genes-associated vasculopathy with onset in infancy; SCA: sickle cell anemia

Funding This research was supported [in part] by the Intramural Research Program of the NIH, NIH 2019 ZIA HL "Vascular remodeling in patients with rare genetic diseases," "Murine models of vascular remodeling," and "iPS-technology and patient specific disease models."

\section{References}

1. Boycott KM, Ardigó D (2018) Addressing challenges in the diagnosis and treatment of rare genetic diseases. Nat Rev Drug Discovery 17:151-152

2. Dawkins HJS, Draghia-Akli R, Lasko P, Lau LPL, Jonker AH, Cutillo CM, Rath A, Boycott KM, Baynam G, Lochmüller H, Kaufmann P, Le Cam Y, Hivert V, Austin CP (2018) Progress in rare diseases research 2010-2016: an IRDiRC perspective. Clin Transl Sci 11:11-20

3. Savoji H, Mohammadi MH, Rafatian N, Toroghi MK, Wang EY, Zhao Y, Korolj A, Ahadian S, Radisic M (2019) Cardiovascular disease models: a game changing paradigm in drug discovery and screening. Biomaterials 198:3-26

4. Mensah GA, Roth GA, Fuster V (2019) The global burden of cardiovascular diseases and risk factors: 2020 and beyond. J Am Coll Cardiol 74:2529-2532

5. Roth GA, Mensah GA, Johnson CO, Addolorato G, Ammirati E et al (2020) Global burden of cardiovascular diseases and risk factors, 1990-2019: update from the GBD 2019 study. J Am Coll Cardiol 76:2982-3021

6. Boycott KM, Rath A, Chong JX, Hartley T, Alkuraya FS, Baynam G, Brookes AJ, Brudno M, Carracedo A, den Dunnen JT, Dyke SOM, Estivill X, Goldblatt J, Gonthier C, Groft SC, Gut I, Hamosh A, Hieter P, Höhn S, Hurles ME, Kaufmann
P, Knoppers BM, Krischer JP, Macek M Jr, Matthijs G, Olry A, Parker S, Paschall J, Philippakis AA, Rehm HL, Robinson PN, Sham PC, Stefanov R, Taruscio D, Unni D, Vanstone MR, Zhang F, Brunner H, Bamshad MJ, Lochmüller H (2017) International cooperation to enable the diagnosis of all rare genetic diseases. Am J Hum Genet 100:695-705

7. Fernandez-Marmiesse A, Gouveia S, Couce ML (2018) NGS technologies as a turning point in rare disease research, diagnosis and treatment. Curr Med Chem 25:404-432

8. Hood L, Tian Q (2012) Systems approaches to biology and disease enable translational systems medicine. Genomics Proteomics Bioinformatics 10:181-185

9. Wu L, Schaid DJ, Sicotte H, Wieben ED, Li H, Petersen GM (2015) Case-only exome sequencing and complex disease susceptibility gene discovery: study design considerations. J Med Genet 52:10-16

10. Lippi M, Stadiotti I, Pompilio G, Sommariva E (2020) Human cell modeling for cardiovascular diseases. Int J Mol Sci 21:6388

11. Schuchardt M, Siegel NV, Babic M, Reshetnik A, Lützenberg R, Zidek W, van der Giet M, Tölle M (2020) A novel long-term ex vivo model for studying vascular calcification pathogenesis: the rat isolated-perfused aorta. J Vasc Res 57:46-52

12. Aiuti A, Biasco L, Scaramuzza S, Ferrua F, Cicalese MP, Baricordi C, Dionisio F, Calabria A, Giannelli S, Castiello MC, Bosticardo M, Evangelio C, Assanelli A, Casiraghi M, Di Nunzio S, Callegaro L, Benati C, Rizzardi P, Pellin D, Di Serio C, Schmidt M, Von Kalle C, Gardner J, Mehta N, Neduva V, Dow DJ, Galy A, Miniero R, Finocchi A, Metin A, Banerjee PP, Orange JS, Galimberti S, Valsecchi MG, Biffi A, Montini E, Villa A, Ciceri F, Roncarolo MG, Naldini L. 2013. Lentiviral hematopoietic stem cell gene therapy in patients with WiskottAldrich syndrome. Science 341

13. St Hilaire C, Ziegler SG, Markello TC, Brusco A, Groden C, Gill F, Carlson-Donohoe H, Lederman RJ, Chen MY, Yang D, Siegenthaler MP, Arduino C, Mancini C, Freudenthal B, Stanescu HC, Zdebik AA, Chaganti RK, Nussbaum RL, Kleta R, Gahl WA, Boehm M (2011) NT5E mutations and arterial calcifications. N Engl J Med 364:432-442

14. Jin H, St Hilaire C, Huang Y, Yang D, Dmitrieva NI, Negro A, Schwartzbeck R, Liu Y, Yu Z, Walts A, Davaine JM, Lee DY, Donahue D, Hsu KS, Chen J, Cheng T, Gahl W, Chen G, Boehm M. 2016. Increased activity of TNAP compensates for reduced adenosine production and promotes ectopic calcification in the genetic disease ACDC. Sci Signal 9: ra121

15. Brofferio A. 2012. Etidronate for arterial calcifications due to deficiency in CD73 (ACDC). clinicaltrials.gov

16. Dmitrieva NI, Walts AD, Nguyen DP, Grubb A, Zhang X, Wang $X$, Ping X, Jin H, Yu Z, Yu ZX, Yang D, Schwartzbeck R, Dalgard CL, Kozel BA, Levin MD, Knutsen RH, Liu D, Milner JD, López DB, O'Connell MP, Lee CR, Myles IA, Hsu AP, Freeman AF, Holland SM, Chen G, Boehm M (2020) Impaired angiogenesis and extracellular matrix metabolism in autosomaldominant hyper-IgE syndrome. J Clin Invest 130:4167-4181

17. Sawada H, Saito T, Nickel NP, Alastalo TP, Glotzbach JP, Chan R, Haghighat L, Fuchs G, Januszyk M, Cao A, Lai YJ, Perez VDJ, Kim YM, Wang L, Chen PI, Spiekerkoetter E, Mitani Y, Gurtner GC, Sarnow P, Rabinovitch M (2014) Reduced BMPR2 expression induces GM-CSF translation and macrophage recruitment in humans and mice to exacerbate pulmonary hypertension. J Exp Med 211:263-280

18. Otsuki S, Saito T, Taylor S, Li D, Moonen J-R, Marciano DP, Harper RL, Cao A, Wang L, Ariza ME, Rabinovitch M. 2021. Monocyte-released HERV-K dUTPase engages TLR4 and MCAM causing endothelial mesenchymal transition. JCI Insight 6 
19. Song S, Yamamura A, Yamamura H, Ayon RJ, Smith KA, Tang H, Makino A, Yuan JX-J (2014) Flow shear stress enhances intracellular $\mathrm{Ca} 2+$ signaling in pulmonary artery smooth muscle cells from patients with pulmonary arterial hypertension. Am J Physiol Cell Physiol 307:C373-C383

20. Kahaleh MB. 2004. Raynaud phenomenon and the vascular disease in scleroderma. Current Opinion in Rheumatology 16

21. Burger D, Touyz RM (2012) Cellular biomarkers of endothelial health: microparticles, endothelial progenitor cells, and circulating endothelial cells. J Am Soc Hypertens 6:85-99

22. Farinacci M, Krahn T, Dinh W, Volk H-D, Düngen H-D, Wagner J, Konen T, von Ahsen O (2019) Circulating endothelial cells as biomarker for cardiovascular diseases. Research and Practice in Thrombosis and Haemostasis 3:49-58

23. Ormiston ML, Toshner MR, Kiskin FN, Huang CJ, Groves E, Morrell NW, Rana AA. 2015. Generation and culture of blood outgrowth endothelial cells from human peripheral blood. $J$ Vis Exp: e53384

24. Theilmann AL, Hawke LG, Hilton LR, Whitford MKM, Cole DV, Mackeil JL, Dunham-Snary KJ, Mewburn J, James PD, Maurice DH, Archer SL, Ormiston ML (2020) Endothelial $<\mathrm{i}>\mathrm{BMPR} 2<$ i $>$ loss drives a proliferative response to BMP (bone morphogenetic protein) 9 via prolonged canonical signaling. Arterioscler Thromb Vasc Biol 40:2605-2618

25. Harper RL, Maiolo S, Ward RJ, Seyfang J, Cockshell MP, Bonder CS, Reynolds PN (2019) BMPR2-expressing bone marrowderived endothelial-like progenitor cells alleviate pulmonary arterial hypertension in vivo. Respirology 24:1095-1103

26. Sen S, McDonald SP, Coates TP, Bonder CS (2011) Endothelial progenitor cells: novel biomarker and promising cell therapy for cardiovascular disease. Clin Science 120:263-283

27. Ormiston ML, Deng Y, Stewart DJ, Courtman DW (2010) Innate immunity in the therapeutic actions of endothelial progenitor cells in pulmonary hypertension. Am J Respir Cell Mol Biol 43:546-554

28. Yoder MC (2009) Defining human endothelial progenitor cells. J Thromb Haemost 7(Suppl 1):49-52

29. Timmermans F, Plum J, Yöder MC, Ingram DA, Vandekerckhove B, Case J (2009) Endothelial progenitor cells: identity defined? J Cell Mol Med 13:87-102

30. Ingram DA, Caplice NM, Yoder MC (2005) Unresolved questions, changing definitions, and novel paradigms for defining endothelial progenitor cells. Blood 106:1525-1531

31. Spitalieri P, Talarico VR, Murdocca M, Novelli G, Sangiuolo F (2016) Human induced pluripotent stem cells for monogenic disease modelling and therapy. World journal of stem cells 8:118-135

32. Chen D, Li Z, Liu Y, Sampaio N, Yang D, Aksentijevich I, Boehm M, Chen G. 2020. Human induced pluripotent stem cells generated from a patient with a homozygous L272P mutation in the OTULIN gene (NIHTVBi014-A). Stem Cell Res 47: 101921

33. Chen G, Li Z, Liu Y, Chen D, Beers J, Cudrici C, Ferrante EA, Schwartzbeck R, Dmitrieva N, Yang D, Zou J, Iruela-Arispe ML, Boehm M. 2020. Generation of human induced pluripotent stem cells (NIHTVBi004-A, NIHTVBi005-A, NIHTVBi006-A, NIHTVBi007-A, NIHTVBi008-A) from 5 CADASIL patients with NOTCH3 mutation. Stem Cell Res 45: 101821

34. Jin H, Yu Z, Navarengom K, Liu Y, Dmitrieva N, Hsu AP, Schwartzbeck R, Cudrici C, Ferrante EA, Yang D, Holland SM, Freeman AF, Boehm M, Chen G. 2019. Generation of human induced pluripotent stem cell lines (NIHTVBi011-A, NIHTVBi012-A, NIHTVBi013-A) from autosomal dominant hyper IgE syndrome (AD-HIES) patients carrying STAT3 mutation. Stem Cell Res 41: 101586

35. Song H-Y, Yang Y-P, Chien Y, Lai W-Y, Lin Y-Y, Chou S-J, Wang M-L, Wang C-Y, Leu H-B, Yu W-C, Chien C-S (2021) Reversal of the inflammatory responses in fabry patient iPSC-derived cardiovascular endothelial cells by CRISPR/Cas9-corrected mutation. Int J Mol Sci 22:2381

36. Yoder MC. 2015. Differentiation of pluripotent stem cells into endothelial cells. Current Opinion in Hematology 22

37. Adams William J, Zhang Y, Cloutier J, Kuchimanchi P, Newton G, Sehrawat S, Aird William C, Mayadas Tanya N, Luscinskas Francis W, García-Cardeña G (2013) Functional vascular endothelium derived from human induced pluripotent stem cells. Stem Cell Reports 1:105-113

38. Peng GY, Lin Y, Li JJ, Wang Y, Huang HY, Shen ZY (2019) The application of induced pluripotent stem cells in pathogenesis study and gene therapy for vascular disorders: current progress and future challenges. Stem Cells Int 2019:9613258

39. Di Bernardini E, Campagnolo P, Margariti A, Zampetaki A, Karamariti E, Hu Y, Xu Q (2014) Endothelial lineage differentiation from induced pluripotent stem cells is regulated by microRNA-21 and transforming growth factor $\beta 2$ (TGF- $\beta 2$ ) pathways*. J Biol Chem 289:3383-3393

40. Sa S, Gu M, Chappell J, Shao NY, Ameen M, Elliott KA, Li D, Grubert F, Li CG, Taylor S, Cao A, Ma Y, Fong R, Nguyen L, Wu JC, Snyder MP, Rabinovitch M (2017) Induced pluripotent stem cell model of pulmonary arterial hypertension reveals novel gene expression and patient specificity. Am J Respir Crit Care Med 195:930-941

41. Gu M, Shao NY, Sa S, Li D, Termglinchan V, Ameen M, Karakikes I, Sosa G, Grubert F, Lee J, Cao A, Taylor S, Ma Y, Zhao Z, Chappell J, Hamid R, Austin ED, Gold JD, Wu JC, Snyder MP, Rabinovitch M (2017) Patient-specific iPSC-derived endothelial cells uncover pathways that protect against pulmonary hypertension in BMPR2 mutation carriers. Cell Stem Cell 20:490504.e5

42. Gu M, Donato M, Guo M, Wary N, Miao Y, Mao S, Saito T, Otsuki S, Wang L, Harper RL, Sa S, Khatri P, Rabinovitch M. 2021. iPSC\&\#x2013; endothelial cell phenotypic drug screening and in silico analyses identify tyrphostin-AG1296 for pulmonary arterial hypertension. Science Translational Medicine 13: eaba6480

43. Tseng W-L, Chou S-J, Chiang H-C, Wang M-L, Chien C-S, Chen K-H, Leu H-B, Wang C-Y, Chang Y-L, Liu Y-Y, Jong Y-J, Lin S-Z, Chiou S-H, Lin S-J, Yu W-C (2017) Imbalanced production of reactive oxygen species and mitochondrial antioxidant SOD2 in Fabry disease-specific human induced pluripotent stem cell-differentiated vascular endothelial cells. Cell Transplant 26:513-527

44. Granata A, Serrano F, Bernard WG, McNamara M, Low L, Sastry P, Sinha S (2017) An iPSC-derived vascular model of Marfan syndrome identifies key mediators of smooth muscle cell death. Nat Genet 49:97-109

45. Zohar B, Blinder Y, Mooney DJ, Levenberg S (2018) Flowinduced vascular network formation and maturation in threedimensional engineered tissue. ACS Biomater Sci Eng 4:1265-1271

46. Kim S, Kim W, Lim S, Jeon JS. 2017. Vasculature-on-a-chip for in vitro disease models. Bioengineering (Basel) 4

47. Chen EP, Toksoy Z, Davis BA, Geibel JP. 2021. 3D bioprinting of vascularized tissues for in vitro and in vivo applications. Frontiers in Bioengineering and Biotechnology 9

48. Zhou X, Nowicki M, Sun H, Hann SY, Cui H, Esworthy T, Lee JD, Plesniak M, Zhang LG (2020) 3D bioprinting-tunable smalldiameter blood vessels with biomimetic biphasic cell layers. ACS Appl Mater Interfaces 12:45904-45915

49. Cui H, Liu C, Esworthy T, Huang Y, Yu ZX, Zhou X, San H, Lee SJ, Hann SY, Boehm M, Mohiuddin M, Fisher JP, Zhang LG. 2020. 4D physiologically adaptable cardiac patch: a 4-month 
in vivo study for the treatment of myocardial infarction. Sci $A d v$ 6: eabb5067

50. Wimmer RA, Leopoldi A, Aichinger M, Kerjaschki D, Penninger JM (2019) Generation of blood vessel organoids from human pluripotent stem cells. Nat Protoc 14:3082-3100

51. Trillhaase A, Aherrahrou Z, Erdmann J. 2021. Abstract 10199: establishing human iPSC-derived vascular organoids as angiogenesis model. Circulation 144: A10199-A

52. Wagstaff PE, Heredero Berzal A, Boon CJF, Quinn PMJ, Ten Asbroek A, Bergen AA. 2021. The role of small molecules and their effect on the molecular mechanisms of early retinal organoid development. Int J Mol Sci 22

53. Borges AC, Broersen K, Leandro P, Fernandes TG. 2022. Engineering organoids for in vitro modeling of phenylketonuria. Frontiers in Molecular Neuroscience 14

54. Mohan SC, Lee T-Y, Giuliano AE, Cui X. 2021. Current status of breast organoid models. Frontiers in Bioengineering and Biotechnology 9

55. Wimmer RA, Leopoldi A, Aichinger M, Wick N, Hantusch B, Novatchkova M, Taubenschmid J, Hämmerle M, Esk C, Bagley JA, Lindenhofer D, Chen G, Boehm M, Agu CA, Yang F, Fu B, Zuber J, Knoblich JA, Kerjaschki D, Penninger JM (2019) Human blood vessel organoids as a model of diabetic vasculopathy. Nature 565:505-510

56. Khan AO, Reyat JS, Bourne JH, Colicchia M, Newby ML, Allen JD, Crispin M, Youd E, Murray PG, Taylor G, Stamataki Z, Richter AG, Cunningham AF, Pugh M, Rayes J. 2021. Stimulation of vascular organoids with SARS-CoV-2 antigens increases endothelial permeability and regulates vasculopathy. medRxiv: 2021.04.25.21255890

57. Monteil V, Kwon H, Prado P, Hagelkrüys A, Wimmer RA, Stahl M, Leopoldi A, Garreta E, Hurtado Del Pozo C, Prosper F, Romero JP, Wirnsberger G, Zhang H, Slutsky AS, Conder R, Montserrat N, Mirazimi A, Penninger JM (2020) Inhibition of SARS-CoV-2 infections in engineered human tissues using clinical-grade soluble human ACE2. Cell 181:905-13.e7

58. Cleaver O (2021) Mouse models of vascular development and disease. Curr Opin Hematol 28:179-188

59. von Scheidt M, Zhao Y, Kurt Z, Pan C, Zeng L, Yang X, Schunkert $\mathrm{H}$, Lusis AJ (2017) Applications and limitations of mouse models for understanding human atherosclerosis. Cell Metab 25:248-261

60. Institute of Medicine Committee on Accelerating Rare Diseases R, Orphan Product D. 2010. The national academies collection: reports funded by National Institutes of Health. In Rare diseases and orphan products: accelerating research and development, ed. MJ Field, TF Boat. Washington (DC): National Academies Press (US) Copyright $\odot$ 2010, National Academy of Sciences.

61. Gold K, Gaharwar AK, Jain A (2019) Emerging trends in multiscale modeling of vascular pathophysiology: organ-on-achip and 3D printing. Biomaterials 196:2-17

62. Kühn R, Schwenk F, Aguet M, Rajewsky K (1995) Inducible gene targeting in mice. Science 269:1427-1429

63. Hong KH, Lee YJ, Lee E, Park SO, Han C, Beppu H, Li E, Raizada MK, Bloch KD, Oh SP (2008) Genetic ablation of the
Bmpr2 gene in pulmonary endothelium is sufficient to predispose to pulmonary arterial hypertension. Circulation 118:722-730

64. West J, Fagan K, Steudel W, Fouty B, Lane K, Harral J, HoedtMiller M, Tada Y, Ozimek J, Tuder R, Rodman DM (2004) Pulmonary hypertension in transgenic mice expressing a dominant-negative BMPRII gene in smooth muscle. Circ Res 94:1109-1114

65. Yona S, Kim K, Wolf Y, Mildner A, Varol D, Breker M, StraussAyali D, Viukov S, Guilliams M, Misharin A, Hume DA, Perlman H, Malissen B, Zelzer E, Jung S (2013) Fate mapping reveals origins and dynamics of monocytes and tissue macrophages under homeostasis. Immunity 38:79-91

66. Alastalo TP, Li M, de Jesus PV, Pham D, Sawada H, Wang JK, Koskenvuo M, Wang L, Freeman BA, Chang HY, Rabinovitch M (2011) Disruption of PPAR $\gamma / \beta$-catenin-mediated regulation of apelin impairs BMP-induced mouse and human pulmonary arterial EC survival. J Clin Investig 121:3735-3746

67. Appleby SL, Mitrofan C, Crosby A, Hoenderdos K, Lodge K, Upton PD, Yates CM, Nash GB, Chilvers ER, Morrell NW (2016) Bone morphogenetic protein 9 enhances lipopolysaccharideinduced leukocyte recruitment to the vascular endothelium. J Immunol 197:3302-3314

68. Yu YA, Malakhau Y, Yu CA, Phelan SJ, Cumming IR, Kan MJ, Mao L, Rajagopal S, Piantadosi CA, Gunn MD. 2020. Nonclassical monocytes sense hypoxia, regulate pulmonary vascular remodeling, and promote pulmonary hypertension. The Journal of Immunology: ji1900239

69. Tojais NF, Cao A, Lai YJ, Wang L, Chen PI, Alcazar MAA, de Jesus Perez VA, Hopper RK, Rhodes CJ, Bill MA, Sakai LY, Rabinovitch M (2017) Codependence of bone morphogenetic protein receptor 2 and transforming growth factor- $\beta$ in elastic fiber assembly and its perturbation in pulmonary arterial hypertension. Arterioscler Thromb Vasc Biol 37:1559-1569

70. Hurst LA, Dunmore BJ, Long L, Crosby A, Al-Lamki R, Deighton J, Southwood M, Yang X, Nikolic MZ, Herrera B, Inman GJ, Bradley JR, Rana AA, Upton PD, Morrell NW (2017) TNF $\alpha$ drives pulmonary arterial hypertension by suppressing the BMP type-II receptor and altering NOTCH signalling 8:14079

71. Feng F, Harper RL, PIN R (2016) BMPR2 gene delivery reduces mutation-related PAH and counteracts TGF- $\beta$-mediated pulmonary cell signalling. Respirology 21:526-532

72. Siedel H, Roers A, Rösen-Wolff A, Luksch H. 2020. Type I interferon-independent T cell impairment in a Tmem173 N153S/ WT mouse model of STING associated vasculopathy with onset in infancy (SAVI). Clinical Immunology 216: 108466

73. Beuzard Y (2008) Mouse models of sickle cell disease. Transfus Clin Biol 15:7-11

74. Salinas Cisneros G, Thein SL. 2020. Recent advances in the treatment of sickle cell disease. Frontiers in Physiology 11

Publisher's Note Springer Nature remains neutral with regard to jurisdictional claims in published maps and institutional affiliations. 